\title{
THE HARNACK INEQUALITY FOR THE RIEMANN-LIOUVILLE FRACTIONAL DERIVATION OPERATOR
}

\author{
RICO ZACHER
}

Abstract. In this note we establish the Harnack inequality for the Riemann-Liouville fractional derivation operator $\partial_{t}^{\alpha}$ of order $\alpha \in(0,1)$. Here the function under consideration is assumed to be globally nonnegative. We show that the Harnack inequality in general fails if this global positivity assumption is replaced by a local one. A Harnack estimate is also derived for nonnegative solutions of a class of nonhomogeneous fractional differential equations.

Mathematics subject classification (2010): 26A33, 45D05, 47G20.

Keywords and phrases: Harnack inequality, fractional derivative, fractional differential equation.

\section{REFERENCES}

[1] R. F. BASS, M. KASSMANn, Harnack inequalities for non-local operators of variable order, Trans. Amer. Math. Soc. 357, 2 (2005), 837-850.

[2] L. CAfFARelli, L. Silvestre, An extension problem related to the fractional Laplacian, Comm. Partial Differential Equations, 32 (2007), 1245-1260.

[3] E. DiBenedetto, Degenerate parabolic equations, Universitext, Springer-Verlag, New York, 1993.

[4] E. DiBenedetto, Partial differential equations, Birkhäuser, 1995.

[5] D. Gilbarg, N. Trudinger, Elliptic partial differential equations of second order, Springer, 1977.

[6] J. Hadamard, Extension à l'équation de la chaleur d'un théorème de A. Harnack, Rend. Circ. Mat. Palermo, 3 (1954), 337-346.

[7] M. Kassmann, Harnack inequalities: an introduction, Bound. Value Probl. 2007, Art. ID 81415, 21 pp.

[8] M. KASSMANN, The classical Harnack inequality fails for non-local operators, preprint.

[9] A. A. Kilbas, H. M. SRivastava, J. J. Trujillo, Theory and applications of fractional differential equations, Elsevier, 2006.

[10] G. M. Lieberman, Second order parabolic differential equations, World Scientific, 1996.

[11] J. Moser, A Harnack inequality for parabolic differential equations, Comm. Pure Appl. Math., 17 (1964), 101-134. Correction in Comm. Pure Appl. Math., 20 (1967), 231-236.

[12] B. PINI, Sulla soluzione generalizzata di Wiener per il primo problema di valori al contorno nel caso parabolico, Rend. Sem. Mat. Univ. Padova, 23 (1954), 422-434.

[13] S. G. SAmko, A. A. Kilbas, O. I. Marichev, Fractional integrals and derivatives. Theory and applications, Gordon and Breach Science Publishers, Yverdon, 1993.

[14] R. ZACHER, A weak Harnack inequality for fractional differential equations, J. Integral Equations Appl., 19 (2007), 209-232.

[15] R. ZACHER, Boundedness of weak solutions to evolutionary partial integro-differential equations with discontinuous coefficients, J. Math. Anal. Appl., 348 (2008), 137-149. 